\title{
Evolution of rotational velocities of A-type stars
}

\author{
Wuming Yang ${ }^{1,2}$, Shaolan $\mathrm{Bi}^{1}$, Xiangcun Meng ${ }^{2}$, Zhijia Tian ${ }^{1}$
}

\begin{abstract}
It was found that the equatorial velocity of A-type stars undergoes an acceleration in the first third of the main sequence (MS) stage, but the velocity decreases as if the stars were not undergoing any redistribution of angular momentum in the external layers in the last stage of the MS phase. Our calculations show that the acceleration and the decrease of the equatorial velocity can be reproduced by the evolution of the differential rotation zero-age MS model with the angular momentum transport caused by hydrodynamic instabilities during the MS stage. The acceleration results from the fact that the angular momentum stored in the interiors of the stars is transported outwards. In the last stage, the core and the radiative envelope are uncoupling, and the rotation of the envelope is a quasi-solid rotation; the uncoupling and the expansion of the envelope lead to that the decrease of the equatorial velocity approximately follows the slope for the change in the equatorial velocity of the model without any redistribution of angular momentum. When the fractional age $0.3 \lesssim \mathrm{t} / \mathrm{t}_{\mathrm{MS}} \lesssim 0.5$, the equatorial velocity remains almost constant for the stars whose central density increases with age in the early stage of the MS phase, while the velocity decreases with age for the stars whose central density decreases with age in the early stage of the MS phase.
\end{abstract}

Subject headings: stars: early-type — stars: rotation — stars: evolution

\section{Introduction}

Helioseismology and asteroseismology have successfully shown us the internal rotation of the Sun and red-giant stars. For example, the Sun has a flat rotational profile (Brown et al. 1989; Kosovichev et al. 1997) but red-giant stars have a fast rotation core (Aerts et al. 2003; Beck et al. 2012), which provided some constraints on the processes of angular momentum transport in the Sun and red-giant stars. In order to reproduce the flat solar rotational profile, the magnetic angular momentum transport (Eggenberger et al. 2005; Yang \& Bi 2006) or the gravity-wave angular momentum transport (Zahn et al. 1997; Talon \& Charbonnel 2005) should be considered in solar models, besides the angular momentum transport caused by hydrodynamic instabilities.

\footnotetext{
${ }^{1}$ Department of Astronomy, Beijing Normal University, Beijing 100875, China; yangwuming@ynao.ac.cn; yangwuming@bnu.edu.cn

${ }^{2}$ School of Physics and Chemistry, Henan Polytechnic University, Jiaozuo 454000, Henan, China
}

Most of A-type stars are known to be fast rotators, with a typical rotational velocity of about 160 $\mathrm{km} \mathrm{s}^{-1}$ (Rover et al. 2007) for stars with masses between about 1.3 and $3.0 \mathrm{M}_{\odot}$. Thus there may be a non-negligible effect of rotation on the structure and evolution of these stars. However, these stars are always hotter than the red edge of the instability strip (Saio \& Gautschy 1998). They are generally not expected to exhibit solar-like oscillations. Therefore the effect of rotation and rotation scenarios of these stars are hard to be understood as that of the Sun and red-giant stars via seismology.

Fortunately, the surface rotational velocities of thousands of stars with the spectral type between late $\mathrm{B}$ and early $\mathrm{F}$ have been observed (Abt \& Morrell 1995; Wolff \& Simon 1997; Rover et al. 2002a, b; Wolff et al. 2004; Rover et al. 2004a b, 2007; Díaz et al.|2011; Zorec \& Rover 2012). These stars include pre-main sequence (PMS) and main sequence (MS) stars. The observed surface rotational velocity of a star depends on: (1) the initial angular momentum, (2) the 
rate of angular momentum loss, (3) the angular momentum transport in stellar interiors, and (4) the changes in the moment of inertia of the star. For stars with masses greater than $1.6 \mathrm{M}_{\odot}$, there is no firm evidence of activity and any significant angular momentum loss during MS evolution (Wolff \& Simon 1997). For these MS stars, thus, the observed surface rotational velocities are mainly dependent on the initial rotational profile at the zero-age main sequence (ZAMS), the angular momentum transport in stellar interiors, and the changes in the moment of inertia of stars. The changes of the moment of inertia can be understood from evolutionary models. Therefore, the observed rotational velocities of A-type stars could provide important clues about the initial rotational profile at the ZAMS and the efficiency of angular momentum transport in stellar interiors.

Recently, Zorec \& Rover (2012) studied the evolution of surface rotational velocities of more than one thousand A-type MS stars. They found that the surface velocities of the stars with masses between about 1.7 and $3.2 \mathrm{M}_{\odot}$ undergo a strong acceleration in the first third of the MS evolutionary phase, which strongly differs from that theoretically predicted by two limiting cases of internal angular momentum redistributions: (i) rigid rotation; (ii) conservation of angular momentum in stellar shells, while in the last third of the MS the velocities decrease as if the stars were not undergoing any redistribution of the angular momentum in the external layers (Zorec \& Royer 2012).

The ZAMS models are always assumed to be a uniform rotation in the calculations of the evolution of rotating stars. If this assumption was correct, a special mechanism might be required to extract angular momentum from the stellar interiors to achieve the acceleration of the equatorial velocity. In fact, Wolff \& Simon (1997) and Wolff et al. (2004) had studied the angular momentum evolution of A- and F-type stars from the birth line to the MS. They concluded that A- and early F-type stars do not loss angular momentum and the angular momentum could be conserved in the shells of the stars as the stars evolve from the end of fully convective phase to the ZAMS. Thus, for these stars the rotation at the ZAMS should be differential (hereafter this differential rotation $Z A M S$ model obtained from the evolution with the conservation of angular momentum in shells was referred to Wolff ZAMS model), and the core could serve as a reservoir of angular momentum which can be transported from the fast rotation core to the slow rotation envelope to produce the acceleration of the equatorial velocity. Moreover, the too high or too low efficiency of angular momentum transport in stellar interiors could not reproduce the acceleration of the surface velocity in the first third of the MS evolutionary phase (Zorec \& Rover 2012). Thus the results of Zorec \& Royer (2012) provide us an opportunity to test the conclusions of Wolff et al. (2004) and the angular momentum transport in the interiors of A-type stars.

In this work, we focus mainly on the evolution of the equatorial velocity of A-type stars. The paper is organized as follow. We simply show our stellar models in section 2 . The results are represented in section 3 . In section 4 , we discuss and summarize the results.

\section{Stellar models and calculation results}

\subsection{Stellar models}

We used the Yale Rotation Evolution Code (Pinsonneault et al. 1989; Yang \& Bi 2007) to compute the evolutions of rotating models with different masses and metallicities. Hydrodynamical instabilities considered in this code are meridional circulation, the Goldreich-SchubertFricke instability, and the secular shear instability (Endal \& Sofia 1978; Pinsonneault et al. 1989). The OPAL EOS tables (Rogers \& Navfonov 2002), OPAL opacity tables (Iglesias \& Rogers 1996), and the opacity tables for low temperature provided by Alexander \& Ferguson (1994) were used. Energy transfer by convection is treated according to the standard mixing length theory. The value of 1.72 for the mixing-length parameter $(\alpha)$ was calibrated against the Sun. The initial metallicity $Z$ was fixed at 0.02 and 0.008 , and the hydrogen abundance was determined by $X=0.767-3 Z$. For a given mass, the initial angular momentum is estimated by using the formula of Kawaler (1987). Angular momentum loss due to magnetic braking and mass loss could be negligible in A-type stars (MacGregor \& Charbonneau 1994; Wolff \& Simon 1997; Zorec \& Rover 2012). Thus we assumed that the total angular momen- 
tum is conserved in our models.

We calculated the following four evolutionary cases: (1) C1, models were evolved from the PMS to the terminal age main sequence (TAMS) without any exchange of angular momentum in shells; (2) $\mathrm{C} 2$, models were computed as a rigid rotator; (3) C3; and (4) C4. In the last two cases, models were evolved from the ZAMS to the TAMS with angular momentum transport caused by the rotationally induced instabilities, and the ZAMS model is a uniform rotator for the $\mathrm{C} 3$ but is a Wolff ZAMS model for the C4. Moreover, we assumed that the rotation of the surface layers (the fractional mass $\delta M / M \simeq 10^{-4}$ ) is uniform.

\subsection{Calculation results}

Figure 1 shows the evolution of the equatorial velocity of models with $M=1.7 \mathrm{M}_{\odot}$ and $Z=0.02$ in the MS life time span $\left(t_{M S}\right)$. The notation $\mathrm{V}_{\mathrm{ZAMS}}$ is the equatorial velocity at the ZAMS. For the cases C1, C2, and C3, from the ZAMS to $\mathrm{t} / \mathrm{t}_{\mathrm{ZAMS}} \approx 0.95$, the equatorial velocities decrease. In case $\mathrm{C} 1$, the equatorial velocity descends about $\mathbf{4 5} \%$, which is consistent with the expectation of the law of conservation of angular momentum in shells ( $V_{e}=V_{0} R_{0} / R_{e}$, where $R_{e}$ is the radius of stars and $V_{0}$ and $R_{0}$ refer to initial values). In the case $\mathrm{C} 2$, the equatorial velocity decreases around $20 \%$. These two limiting cases did not reproduce the acceleration of the surface velocity in the early stage of MS. The evolution of the equatorial velocity of case C3 is similar to that of the case C2 except for in the last stage of the MS. This is because the rotation of the radiative region of the MS models of the C3 is a quasi-solid body rotation and the core and the envelope are uncoupling in the last stage. In the case C4, the equatorial velocity undergoes an acceleration in the early stage of the $\mathrm{MS}$ phase. From $\mathrm{t} / \mathrm{t}_{\mathrm{MS}} \simeq 0.017$ to $\mathrm{t} / \mathrm{t}_{\mathrm{MS}} \simeq 0.3$, the equatorial velocity increases about $20 \%$. But when $\mathrm{t} / \mathrm{t}_{\mathrm{MS}}$ increases from about 0.3 to 0.5 , the velocity remains almost constant. After $\mathrm{t} / \mathrm{t}_{\mathrm{MS}}>$ 0.5 , the velocity decreases as evolution proceeds until the evolution approaches the end of the MS stage. When the fractional age $t / t_{M S}>0.7$, the decrease of the equatorial velocity of the case $\mathrm{C} 4$ is similar to that of the case $\mathrm{C} 1$, which is consistent with the finding of Zorec \& Rover (2012).

Figure 2 shows the radial distributions of internal angular velocity of the model with $M=$
$1.7 \mathrm{M}_{\odot}$ at different stages of the evolution of case C4. Because there is no redistribution of angular momentum in shells during the PMS stage and the core of the model contracts but the envelope expands, the core rotates faster than the envelope when the model evolves to the ZAMS. From $\mathrm{t} / \mathrm{t}_{\mathrm{MS}} \simeq 0.017$ to $\mathrm{t} / \mathrm{t}_{\mathrm{MS}} \simeq 0.30$, the angular momentum stored in the fast rotation core is transported to the slow rotation envelope, which almost fully impedes the decrease in the angular velocity of the envelope, even leads to an increase in the angular velocity. In addition, due to the increase in radius, the equatorial velocity increases obviously. When the model evolves from $\mathrm{t} / \mathrm{t}_{\mathrm{MS}} \approx$ 0.3 to $\mathrm{t} / \mathrm{t}_{\mathrm{MS}} \approx 0.5$, the core and the envelope are strongly coupled by hydrodynamic instabilities. Although the core still contracts, its angular velocity decreases with the decrease in the angular velocity of the envelope. The angular momentum stored in the stellar interiors is continually transported outwards, which is insufficient to remain the acceleration of the equatorial velocity but can allow the velocity to remain almost constant. When $t / t_{\mathrm{MS}}>0.6$, due to the facts that the core contracts and the envelope expands rapidly, the hydrodynamical instabilities are insufficient to couple the core to the envelope. Thus the core and the envelope are uncoupling. However, the instabilities are sufficient to keep the envelope rotating as a quasi-solid body. In our models, the differential rotation results from the contraction/expansion of stars. Due to the uncoupling of the core and the envelope, the decrease of the angular velocity of the envelope depends almost only on the expansion of the envelope. Moreover, Fig. 3 shows that most of the radiative region spin down at an approximately equal rate from $\mathrm{t} / \mathrm{t}_{\mathrm{MS}} \approx 0.7$ to $\mathrm{t} / \mathrm{t}_{\mathrm{MS}} \approx 0.9$ in the evolution of case $\mathrm{C} 1$, which is similar to the manner of quasi-solid rotation. Thus when the fractional age $\mathrm{t} / \mathrm{t}_{\mathrm{MS}}>0.7$, the decrease in the equatorial velocities of cases $\mathrm{C} 1$ and C4 follows an approximate slope.

We also calculated the evolutions of the equatorial velocity of models with $M=\mathbf{2 . 0}$, 2.5, and $3.0 \mathrm{M}_{\odot}$. The comparisons of the evolutions of the equatorial velocities of our models with the results of Zorec \& Rover (2012) are shown in Fig. 4, The evolutions of cases $\mathrm{C} 1, \mathrm{C} 2$, and $\mathrm{C} 3$ of these models are similar to those of model with $M=1.7 \mathrm{M}_{\odot}$, i.e. the equa- 
torial velocity decreases with age. But in the early stage $\left(\mathrm{t} / \mathrm{t}_{\mathrm{MS}} \lesssim 0.3\right)$ of the MS of the case $\mathrm{C} 4$, the equatorial velocity undergoes an acceleration. However, the magnitude of the increase of the ratio $V_{e} / V_{\text {ZAMS }}$ of our models is obviously lower than that obtained by Zorec \& Royer (2012). For example, at $t / t_{M S}=0.35$, the value of the observed $V_{e} / V_{Z A M S}$ is $\mathbf{1 . 2 6 5} \pm \mathbf{0 . 0 7 9}$ for the star with $M=2.5 \mathrm{M}_{\odot}$, but the value for our models is about 1.11. The discrepancy is about $2 \sigma$. When the fractional age $\mathrm{t} / \mathrm{t}_{\mathrm{MS}}$ increases from around 0.3 to 0.5 , the theoretical velocities decrease slightly but the observed ones decrease rapidly for stars with $M=\mathbf{2 . 5}$ and $3.0 \mathrm{M}_{\odot}$. When the age $\mathrm{t} / \mathrm{t}_{\mathrm{MS}}>0.5$, the value of the theoretical $\mathrm{V}_{\mathrm{e}} / \mathrm{V}_{\mathrm{ZAMS}}$ is lower for the star with $M=2.0 \mathrm{M}_{\odot}$ but is higher for the star with $M=3.0 \mathrm{M}_{\odot}$ than the observed one. However, for the star with $M=2.5 \mathrm{M}_{\odot}$, the evolution of the theoretical $\mathrm{V}_{\mathrm{e}} / \mathrm{V}_{\mathrm{ZAMS}}$ is good agreement with the observed one. After $t / t_{M S}>0.7$, the decrease of the velocities of cases $\mathrm{C} 1$ and $\mathrm{C} 4$ and the observed one follow an approximate slope.

Furthermore, the Fig. 4 shows that the evolution of the equatorial velocities is slightly affected by the metallicity. This can be due to the fact that the radius of low-Z model is smaller than that of high- $Z$ model.

\section{Discussion and Conclusions}

The increase of the fractional velocity $\mathrm{V}_{\mathrm{e}} / \mathrm{V}_{\mathrm{ZAMS}}$ is mainly dependent on the angular momentum that is stored in the interiors of the star and can be transported outwards to accelerate the surface layers. The angular momentum depends on the contraction of the stellar core and the expansion of the envelope of the star in our models. The more massive the star, the less the contraction of its core when the star evolves from the PMS to the ZAMS. In addition, in the early stage of the MS, the central density increases as the evolution proceeds for the stars with $M \lesssim 2.0 \mathrm{M}_{\odot}$, but the central density decreases (see the Fig. 5) for the stars with $M \gtrsim 2.1 \mathrm{M}_{\odot}$, i.e. the angular velocity of the core decreases for stars with $M \gtrsim 2.1$ $\mathrm{M}_{\odot}$. As a consequence, the more massive the star, the smaller the fractional angular momentum that can be transported outwards. Moreover, the more massive the star, the larger the expansion of the radius during the MS stage, thus the more difficult to remain the angular velocity of the surface layer. Therefore the increase of the fractional velocity decreases with increasing the mass, and the available angular momentum stored in the stellar interiors is almost drained when $\mathrm{t} / \mathrm{t}_{\mathrm{MS}} \approx 0.3$ for the stars with $M>2.1 \mathrm{M}_{\odot}$. Hence from $\mathrm{t} / \mathrm{t}_{\mathrm{MS}} \approx 0.3$ to $\mathrm{t} / \mathrm{t}_{\mathrm{MS}} \approx 0.5$, the equatorial velocity decreases for stars with $M>2.1 \mathrm{M}_{\odot}$.

The instabilities caused that the angular momentum was transported outwards in the early stage of the MS, which leads to the increase in the $V_{e} / V_{Z A M S}$. Although the magnitude of the increase in the fractional velocity of our models is less than that obtained by Zorec \& Rover (2012), who given the magnitude as high as $30 \%$, the velocity increases as the evolution proceeds during the first third of the MS phase, which is consistent with the finding of Zorec \& Royer (2012). This might offer a support for the Wolff ZAMS model and imply that the core and the envelope is uncoupling as A-type stars evolve from the end of fully convective phase to the ZAMS but is coupling in the early stage of the MS. The theoretical magnitude is lower than the observed one. This might be caused by that the efficiency of angular momentum transport wasn't calibrated.

In the last stage of the MS $\left(0.7 \lesssim \mathrm{t} / \mathrm{t}_{\mathrm{MS}} \lesssim 0.95\right)$, the core and the radiative envelope are decoupling in the evolution of cases $\mathrm{C} 1, \mathrm{C} 3$, and $\mathrm{C} 4$. The contraction of the core almost not affect the rotation of the envelope. For the case C1, although the rotation of the whole radiative envelope is differential, the envelope spins down at an approximately equal rate in our models, which is similar to the manner of the quasi-solid body rotation. For the case $\mathrm{C} 4$, the rotation of the radiative envelope is a quasi-solid rotation. Thus the decrease of the fractional velocity of the cases $\mathrm{C} 1$ and $\mathrm{C} 4$ follows an approximate slope when $t / t_{M S} \gtrsim 0.7$. As a consequence, although the velocities of A-type stars decrease as if the stars were not undergoing any redistribution of the angular momentum in their envelope during the final stages of the MS phase, the rotation of the envelope of A-type stars might be a quasi-solid rotation with the decoupling of 
the core and the envelope. Thus, the rotation of A-type stars might be closer to the quasisolid body rotation during $t / t_{\mathrm{MS}} \approx 0.3-0.5$ than in the early or last stage of the MS.

Compared with Ekström et al. (2008) models used by Zorec \& Rover (2012), our ZAMS models for C4 are the Wolff ZAMS model but the rotation of the ZAMS models of Ekström et al. (2008) and our C3 is a solid body rotation. The equatorial velocity of our C3 decreases with age, which is similar to that obtained by Zorec \& Rover (2012) from Ekström et al. (2008) models. The cases $\mathrm{C} 3$ and $\mathrm{C} 4$ have the same instabilities, this may imply that the Wolff ZAMS model is required to achieve the acceleration. Moreover, we neglected the angular momentum loss caused by mass loss. Finally, there are some differences in the treatments of instabilities included models (Pinsonneault et al. 1989; Maeder \& Zahn 1998), which can lead to the differences in the efficiency of angular momentum transport and the mixing of elements. Besides the hydrostatic and Von Zeipel effects, the mixing caused by rotationally induced instabilities is one of the key factors that affects the distribution of density and the instability of convection by changing the distribution of elements. In our models, the effects of rotation lead to a decrease in the convective core for stars with $M \lesssim$ $2.0 \mathrm{M}_{\odot}$ but an increase in the convective core for stars with $M \gtrsim \mathbf{2 . 1} \mathrm{M}_{\odot}$ (Yang et al. 2012). As a consequence, near the end of the MS, the radius of rotating models is smaller than that of non-rotating ones for the stars with $M \lesssim \mathbf{2 . 0} \mathrm{M}_{\odot}$ but larger than that of non-rotating models for the stars with $M \gtrsim 2.1 \mathrm{M}_{\odot}$. Due to the fact that the decrease of the ratio $V_{e} / V_{Z A M S}$ is relevant to the increase in the radius, thus the instabilities make the stars with $M \lesssim \mathbf{2 . 0} \mathrm{M}_{\odot}$ easier remain their $V_{\mathrm{e}} / \mathrm{V}_{\mathrm{ZAMS}}$ at a high value than the stars with $M \gtrsim 2.1 \mathrm{M}_{\odot}$.

In this work, we calculated the evolution of the equatorial velocity of A-type stars with different conditions. The evolution of the case $\mathrm{C} 4$ reproduces the most characteristics of the equatorial velocity of A-type stars that were found by Zorec \& Rover (2012). From the ZAMS to $\mathrm{t} / \mathrm{t}_{\mathrm{MS}} \approx 0.3$, the equatorial velocity undergoes an acceleration. But in the last stage of the MS stage $\left(\mathrm{t} / \mathrm{t}_{\mathrm{MS}} \gtrsim 0.7\right)$, the core and the envelope of $\mathrm{A}$ type stars are uncoupling; the envelope rotates as a quasi-solid body, but the decrease of the equatorial velocity approximately follows the slope of the velocity of the model without any redistribution of the angular momentum in this stage. However, the magnitude of the increase of the fractional velocity $\mathrm{V}_{\mathrm{e}} / \mathrm{V}_{\mathrm{ZAMS}}$ of our models is less than that obtained by Zorec \& Royer (2012). Moreover, our models with $M=\mathbf{2 . 5}$ and $3.0 \mathrm{M}_{\odot}$ did not reproduce the rapid decrease in the equatorial velocity during the middle stage of the MS phase. This might imply that some mechanisms for the transport or loss of angular momentum are missing in our models.

We thank the anonymous referee for his/her helpful comments and J. Zorec and F. Royer for providing their data and acknowledge the support from the NSFC 11273012, 11273007, 10933002, and 11003003, and the Project of Science and Technology from the Ministry of Education (211102). 


\section{REFERENCES}

Abt, H. A., \& Morrell, N. I. 1995, ApJS, 99, 135

Alexander, D. R., \& Ferguson, J. W. 1994, ApJ, 437,846

Aerts, C. et al. 2003, Science, 300, 1926

Beck, P. G. et al. 2012, Nature, 481, 55

Brown, T. M., Christensen-Dalsgaard, J., Dziembowski, W. A., Goode, P., Gough, D. O., \& Morrow, C. A. 1989, ApJ, 343, 526

Díaz, C. G., González, J. F., Levato, H., \& Grosso, M. 2011, A\&A 531, A143

Eggenberger, P., Maeder, A., \& Meynet, G. 2005, A\&A, 440, L9

Ekström, S., Meynet, G., Maeder, A., \& Barblan, F. 2008, A\&A, 478, 467

Endal, A. S., \& Sofia, S. 1978, ApJ, 220, 279

Iglesias, C., \& Rogers, F. J. 1996, ApJ, 464, 943

Kawaler, S. D. 1987, PASP, 99, 1322

Kosovichev, A. G. et al. 1997, Sol. Phys., 170, 43

MacGregor, K. B., \& Charbonneau, P. 1994, ASPC, 64, 174

Maeder, A., \& Zahn, J. P. 1998, A\&A, 334, 1000

Pinsonneault, M. H., Kawaler, S. D., Sofia, S., \& Demarqure, P. 1989, ApJ, 338, 424

Rogers, F. J., \& Nayfonov, A. 2002, ApJ, 576,1064

Royer, F., Gerbaldi, M., Faraggiana, R., \& Gómez, A. E. 2002a, A\&A, 381, 105

Royer, F., Grenier, S., Baylac, M. O., Gómez, A. E., \& Zorec, J. 2002b, A\&A, 393, 897

Royer, F., Zorec, J., \& Frémat, Y. 2004a, IAUS, 215,55

Royer, F., Zorec, J., \& Gómez, A. E. 2004b, IAUS, 224, 109

Royer, F., Zorec, J., \& Gómez, A. E. 2007, A\&A, 463,671

Saio, H., \& Gautschy, A. 1998, ApJ, 498, 360
Talon, S., \& Charbonnel, C. 2005, A\&A, 440, 981

Wolff, S. C., \& Simon, T. 1997, PASP, 109, 759

Wolff, S. C., Strom, S. E., \& Hillenbrand, L. A. 2004, ApJ, 601, 979

Yang, W. M., \& Bi, S. L. 2006, A\&A, 449, 1161

Yang, W. M., \& Bi, S. L. 2007, ApJ, 658, L67

Yang, W. M., \& Bi, S. L. 2008, Chjaa, 8, 677

Yang, W., Bi, S., \& Meng, X. 2012, eprint arXiv:1212.1511

Zahn, J. P., Talon, S., \& Matias, J. 1997, A\&A, 322,320

Zorec, J., \& Royer, F. 2012, A\&A, 537, A120

This 2-column preprint was prepared with the AAS IATEX macros v5.2. 


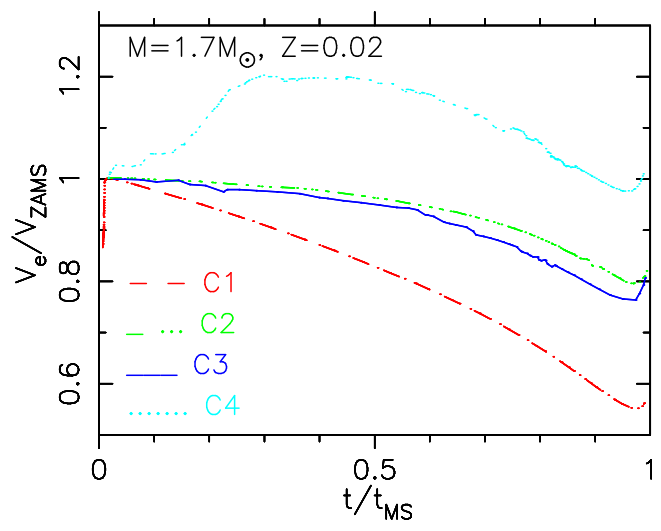

Fig. 1.- Evolution of the equatorial velocity in the MS stage. The long-dashed (red) line shows the evolution of $\mathrm{C} 1$. The dash-triple-dotted (green) line indicates the result of $\mathrm{C} 2$. The solid (blue) line is given for C3. The dotted (cyan) line corresponds to the evolution of $\mathrm{C} 4$.

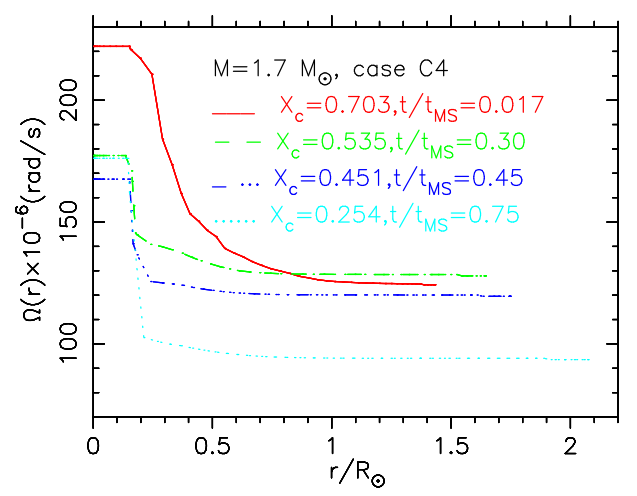

Fig. 2.- The radial distributions of internal angular velocity at different evolutionary stages. The solid (red) line shows the distribution at $X_{c}=0.703$. The long-dashed (green) line indicates the distribution at $X_{c}=0.535$. The dashtriple-dotted (blue) line shows the distribution at $X_{c}=0.451$, and the dotted (cyan) line refers to the distribution at $X_{c}=0.254$.

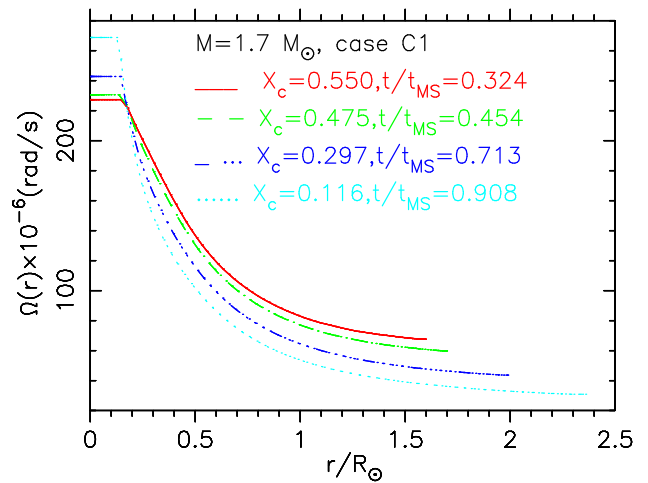

Fig. 3.- Same as Fig. 2 but for the evolutions of case $\mathrm{C} 1$.
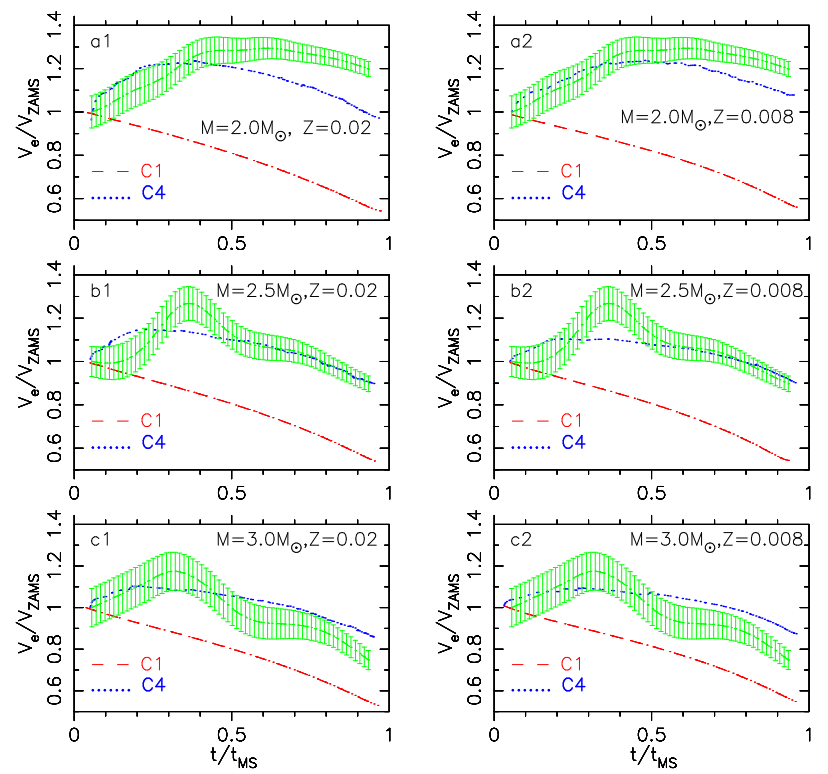

Fig. 4.- Comparisons of the evolutions of theoretically equatorial velocities with the observed data. The dash-dotted lines (green) show the results of Zorec \& Rover (2012). The error bars (green) represent $1 \sigma$ errors. 

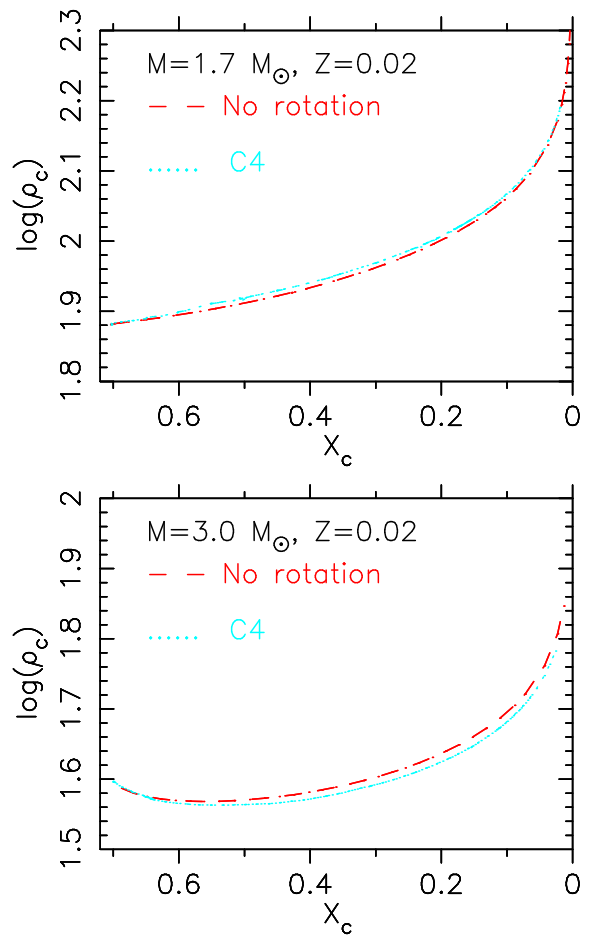

Fig. 5.- The central density $\left(\rho_{c}\right)$ as a function of the mass fraction of central hydrogen. The dashed (red) lines show the results of models without rotation. The dotted (cyan) lines indicate the results of models with rotation. 